\title{
La diversité génétique des bananiers plantains cultivés dans la zone Ouest de la Région des Plateaux au Togo
}

\author{
K. ODAH ${ }^{*}$, M. AZIADEKEY ${ }^{2}$, K. TOZO ${ }^{1}$, S. AKPAVI ${ }^{3}$, R. KOUKOUMA ${ }^{2}$, \\ A. GUELLY ${ }^{3}, \mathrm{~K}_{\text {. KOKOU }}{ }^{3}, \mathrm{~K}_{\text {. ASSIGNON }}{ }^{4}, \mathrm{Y}$. AKOGO ${ }^{1}$, A. AIDAM ${ }^{1}$, \\ K. AKPAGANA ${ }^{3}$, L. KENNY ${ }^{5}$ A. AIT-OUBAHOU ${ }^{5}{\text { C. } \text { ZINSOU }^{6} \text { et M. GBEASSOR }}^{7}$ \\ ${ }^{I}$ Laboratoire de Physiologie et Biotechnologie Végétales, Faculté des Sciences, \\ Université de Lomé, BP : 1515 Lomé, Togo. \\ ${ }^{2}$ Département de Génétique et d'Amélioration des plantes, Ecole Supérieure d'Agronomie, \\ Université de Lomé, BP : 1515, Lomé, Togo. \\ ${ }^{3}$ Laboratoire de Botanique et d'Ecologie Végétale, Faculté des Sciences, \\ Université de Lomé BP : 1515 Lomé, Togo. \\ ${ }^{4}$ Laboratoire de Phytopathologie et de Biotechnologies Végétales ITRA/CRAL, BP 2318 Lomé, Togo. \\ ${ }^{5}$ Institut Agronomique et Vétérinaire Hassan II, Complexe Horticole d'Agadir, Maroc. \\ ${ }^{6}$ Université des Antilles-Guyane, Poîte-à-Pitre, Guadeloupe. \\ ${ }^{7}$ Laboratoire de Pharmacologie-Physiologie, Faculté des Sciences, \\ Université de Lomé, BP : 1515 Lomé, Togo. \\ *Auteur correspondant, E-mail : komiodah@hotmail.com
}

\section{RESUME}

Le bananier représente l'une des cultures les plus importantes dans le monde. Au Togo, la culture de bananiers plantains se fait surtout dans la Région des Plateaux à l'Ouest dans de petites exploitations agricoles ; ce qui a entrainé une faible productivité. Cependant, les variétés de bananiers plantains cultivées ne sont pas bien connues. Un inventaire a été entrepris dans le but de dénombrer les variétés de bananiers plantains cultivés grâce à des paramètres agromorphologiques et aussi de montrer l'intérêt que suscite la valorisation de la culture de cette plante. Au terme de cette étude, cinq (5) variétés de bananiers plantains ont été recensées. La variété 'Apim' est la plus productive suivie de la variété ‘Abladzo'. Contrairement aux autres, la variété 'Taévé' produit 2 régimes par pied au lieu d'un régime. Le cycle végétatif des bananiers plantains varie de 11 à 15 mois sauf pour la variété 'Apim' qui est de 18 mois. Des études de propagation rapide doivent être envisagées pour la sélection des variétés hautement performantes et la production massive du plantain pour les populations locales.

() 2013 International Formulae Group. All rights reserved.

Mots clés : Togo, bananier plantain, diversité génétique.

\section{INTRODUCTION}

Le bananier représente l'une des cultures les plus importantes dans le monde. Il est originaire de l'Asie du Sud-Est (Bakry et al., 2002) et cultivé dans plus de 120 pays des zones intertropicales (Jones, 2000). Ils appartiennent à la classe des
Monocotylédones, de l'ordre des Scitaminales et à la famille des Musacées. On en distingue deux genres : le genre Musa et le genre Ensete (Champion, 1963). Les espèces de bananier les plus cultivées appartiennent au genre Musa. Ce sont essentiellement Musa accuminata et Musa balbisiana (Bakary et al., 
2002). Ces deux espèces sont à l'origine des divers cultivars classés sur la base de leurs caractères morphologiques et de leur constitution génétique. Il existe actuellement plus de 500 variétés de bananiers comestibles (Perrier et Tezenas, 1990). D'un point de vue culinaire, on distingue 2 types de bananes : les bananes à dessert, comme celles que nous mangeons au petit-déjeuner, et les bananes à cuire ou bananes plantains.

La banane plantain constitue un aliment de base pour les populations des zones de production (Gire, 1994). Elle occupe le $4^{\text {ème }}$ rang des produits vivriers en termes de consommation après le blé, le maïs et le riz (FAO, 2009). C'est la principale source alimentaire pour plus de 400 millions de personnes à travers le monde et particulièrement dans les pays tropicaux (Jones, 2000). La plante représente aussi une source substantielle de revenus pour de nombreuses populations rurales et urbaines. Elle est généralement produite en culture de case ou en association avec d'autres cultures (l'igname, le manioc, l'aubergine, etc.). L'association est la principale forme de production de la banane plantain (Nkendah et Akyeampong, 2003). La monoculture, rarement pratiquée est effectuée sur de petites surfaces. La plupart des cultivars de bananier produit des fruits aspermes. Leur propagation se fait à partir de rejets issus de la plante mère et qui constituent le matériel de plantation classique.

Le bananier est une plante herbacée dont la taille varie de 1,5 à 8 mètres selon les espèces et les cultivars. Le fruit, la banane est riche en antioxydants, et sa consommation préviendrait l'apparition de nombreuses maladies comme le cancer, l'ulcère d'estomac, diabète de type 2, etc. (Anonyme, 2013). De plus, les sucres qu'elle contient contribueraient à maintenir une bonne santé gastro-intestinale. C'est une excellente source de vitamines (C, B6, B9), de minéraux (potassium, magnésium, cuivre) et a des bienfaits sur la santé de l'Homme (INIBAP, 2005). Outre le fruit, le pseudo tronc du bananier est utilisé dans la fabrication de pâte à papier, de cordages, de tissus, de vêtements et de filets de pêche. Les feuilles servent d'assiette, le bourgeon mâle est cuit comme légume et les racines servent en décoction médicinale. Aussi, la sève est utilisée comme encre indélébile et les fibres de la tige constituent un absorbant de haute porosité et capillarité ; ils sont utilisés dans des systèmes naturels de purification de l'eau ou pour l'absorption de l'huile (CIRAD, 2001; Anirudhan et Shibi, 2007). Cependant, peu de recherches ont été consacrées au bananier. Cela s'explique en partie par la biologie de la plante. En effet, la plupart des variétés indigènes comestibles sont des triploïdes stériles qui ont un cycle de vie allant d'un à trois ans. Dans ces conditions, les programmes d'hybridation ont de grandes difficultés pour aboutir et des travaux de recherche ont été très peu entrepris. Néanmoins, des études récentes ont été menées sur l'innovation de nouvelles techniques et l'amélioration de la culture des plantains en Afrique de l'Ouest et Centrale par Koné et al. (2010) et Tomékpé et al. (2011) pour augmenter sa production.

$\mathrm{Au}$ Togo, la culture des bananiers ne suscite pas beaucoup d'intérêt chez les paysans. Ils sont souvent cultivés dans de petites exploitations, dans des jardins de case, dans des enclos ou servent de clôture à des habitations. Les bananiers ne sont cultivés que dans la région des plateaux à cause du sol qui est favorable à sa culture. Cette production bananière est presque destinée à la consommation locale et reste d'ailleurs insuffisante à la demande. Il est donc utile d'identifier les différentes variétés existantes de bananiers et de travailler sur leur diversité génétique pour augmenter leur production comme l'a noté Lescot (2007).

Le but du présent travail est de faire l'inventaire des différentes variétés de bananiers plantains cultivées au Togo grâce à des descripteurs morpho botaniques, d'identifier la diversité génétique au sein de ce groupe et de montrer l'intérêt que suscite la valorisation de la culture de cette plante. Elle représente une énorme source d'alimentation et de richesse pour de nombreuses populations locales pauvres qui en ont besoin pour leur survie dans un contexte de paupérisation généralisée. 


\section{MATERIEL ET METHODES}

\section{Zone d'étude}

L'étude a été effectuée dans les préfectures de Kloto, de Wawa et d'Akébou qui sont toutes situées dans la zone ouest de la région des plateaux (Figure 1) du Togo. Ces préfectures constituent la principale zone de culture du bananier du pays. La zone est caractérisée par un climat tropical humide (Brabant et al., 1996) et des terres riches en matières organiques. La pluviométrie annuelle se situe entre 1400 à $1800 \mathrm{~mm}$ avec une température moyenne variant entre 21 et 28 ${ }^{\circ} \mathrm{C}$.

\section{Organisation de l'étude}

Les différents bananiers cultivés ont été recensés en utilisant les descripteurs proposés par IPGRI-INIBAP/CIRAD (1996). Une attention particulière a été portée sur la morphologie de la plante, au régime et aux fruits. Nous avons réalisé la description agromorphologique du plantain et du bananier en nous basant sur la taille du pseudo-tronc, l'inhibition des rejets, les feuilles, le nombre de mains par régime, le nombre de doigts par main, la longueur des doigts, la couleur de la peau du doigt ou de la banane mûre, la taille et la grosseur de la banane, la forme du fruit et l'apex du fruit. Au cours de cette enquête, quarante-huit (48) producteurs de bananes répartis dans douze villages de la zone d'étude ont été visités.

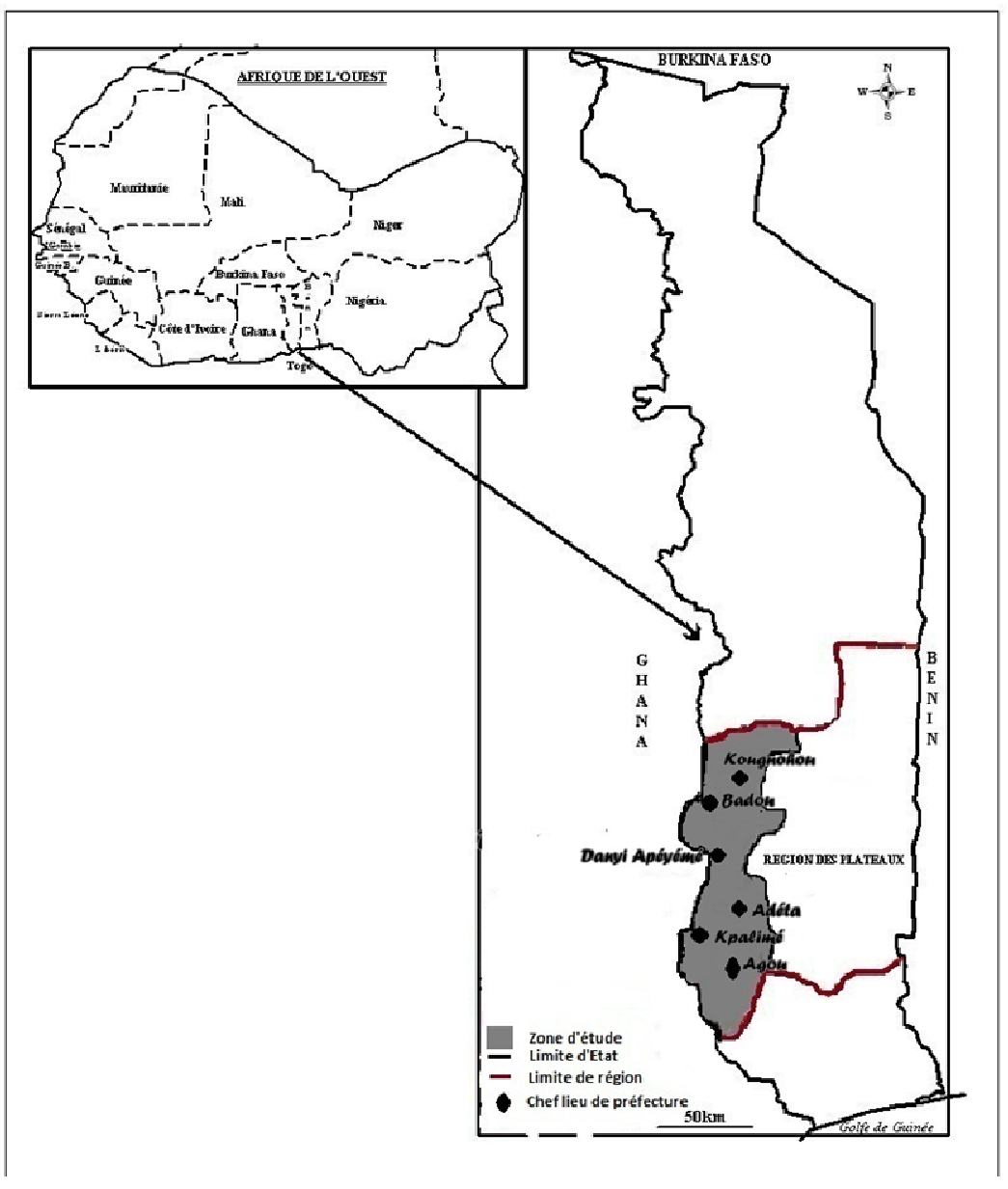

Figure 1 : Carte du Togo montrant la zone d'étude. 


\section{RESULTATS}

$\mathrm{Au}$ terme de cette étude nous avons inventorié cinq (5) variétés de bananiers plantains (Tableau 1). Ils sont beaucoup plus cultivés dans les plantations que dans des jardins de case. Ils constituent un groupe homogène important du point de vue morphologique. Les bananiers plantains possèdent un pseudo-tronc inférieur ou égal à $7 \mathrm{~m}$ avec une inhibition plus ou moins forte des rejets. Chez la plupart des bananiers plantains rencontrés, on note une forte baisse du nombre des feuilles fonctionnelles à la floraison. Les bananiers plantains produisent des fruits «bananes» qui sont des baies parthénocarpiques formées sans fécondation ne contenant donc pas de graines (si on fend cette «banane domestique » dans le sens de la longueur, on observe une série longitudinale de petits points noirs qui sont des ovules non fécondés). Le fruit est long, légèrement incurvé et regroupé sur le bananier en grappes nommées « régimes ». Les bananes sont cueillies vertes dans les plantations et possède une peau difficile à détacher. La partie intérieure est une pulpe amylacée, rosâtre au goût aigre, mais elle est généralement épaisse et sucrée quand le fruit est mûr (Figure 2).

La variété 'Apim' (Figure 3) est la plus productive des bananiers plantains rencontrés. En effet, elle peut produire jusqu'à 288 doigts par régime. Cette variété est cultivée dans la même zone que la variété 'Abladzo' qui ne produit que 50 doigts par régime. Cette dernière variété est en voie de disparition à cause de sa faible rentabilité pour les paysans. Les variétés de plantains inventoriées possèdent en général une faible productivité par rapport aux bananiers doux. Elles possèdent également, en dehors de la variété 'Apim', une plus faible productivité par rapport à la variété intermédiaire représentée par 'Abidjankodu' qui produits 130 fruits par régime (Odah et al., 2012). Toutes les variétés de plantains produisent un seul régime par pied sauf la variété 'Taévé' (Figure 4) qui produit 2 régimes par pied d'où son nom qui signifie « 2 têtes » en langue locale «Ewé ». Chaque régime porte 5 mains dont chacune possède 6 doigts. Le cycle végétatif des plantains varie de 11 à 15 mois sauf pour la variété 'Apim' qui est de 18 mois.

La banane à cuire ou banane plantain est l'aliment de base des populations dans les zones de production du Togo. Toutes les bananes à cuire vendues sur les marchés de Lomé proviennent de la zone prospectée. Il existe cependant des bananiers dans d'autres zones du pays cultivés sous forme de jardins de case et dont les fruits sont destinés à la consommation locale.

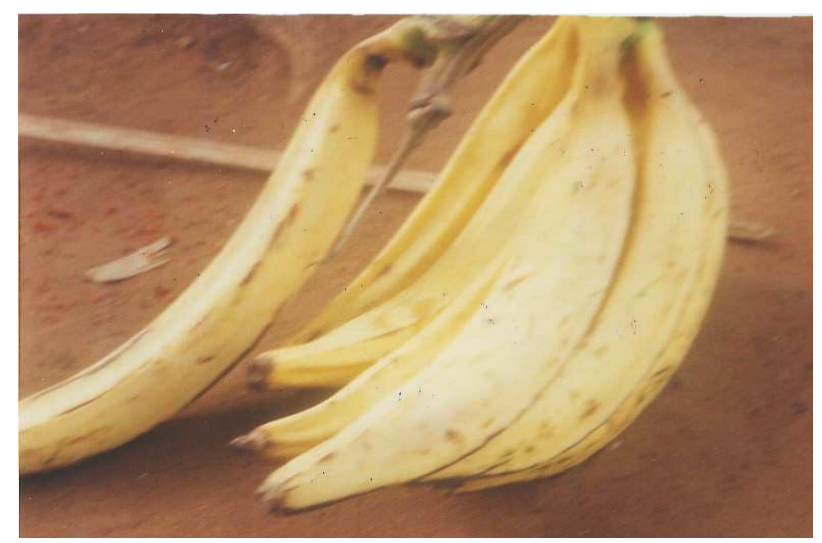

Figure 2: Un régime de Agbavé portant 2 mains dont une a un doigt et l'autre 6 doigts mûrs. 


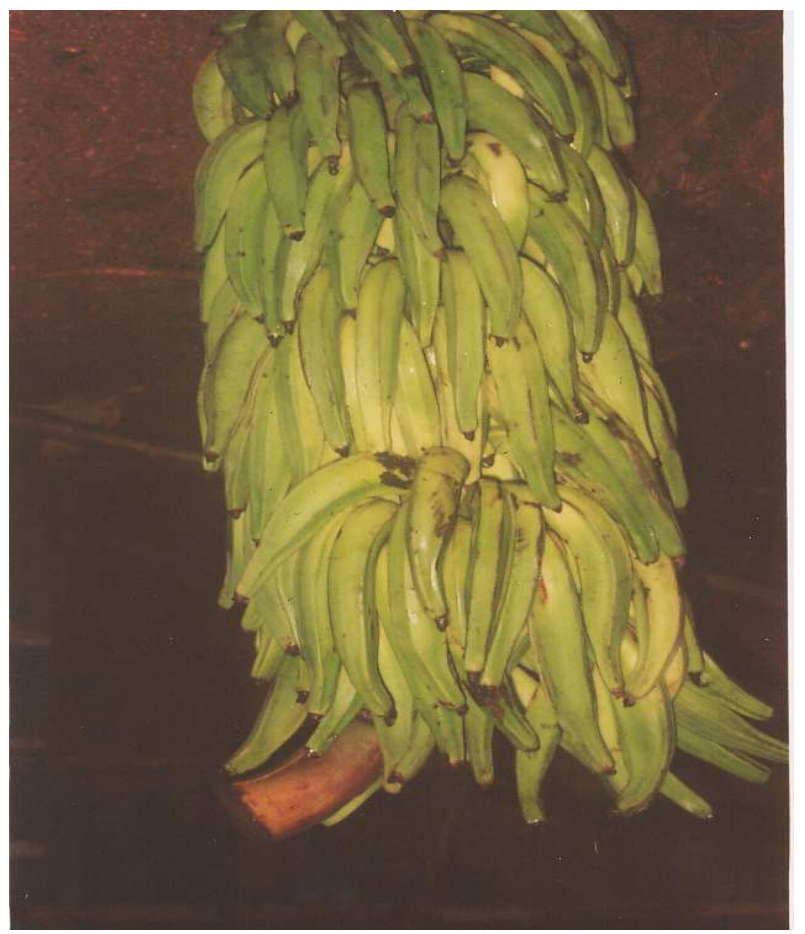

Figure 3 : Un régime d'Apim avec plusieurs mains portant plusieurs doigts.

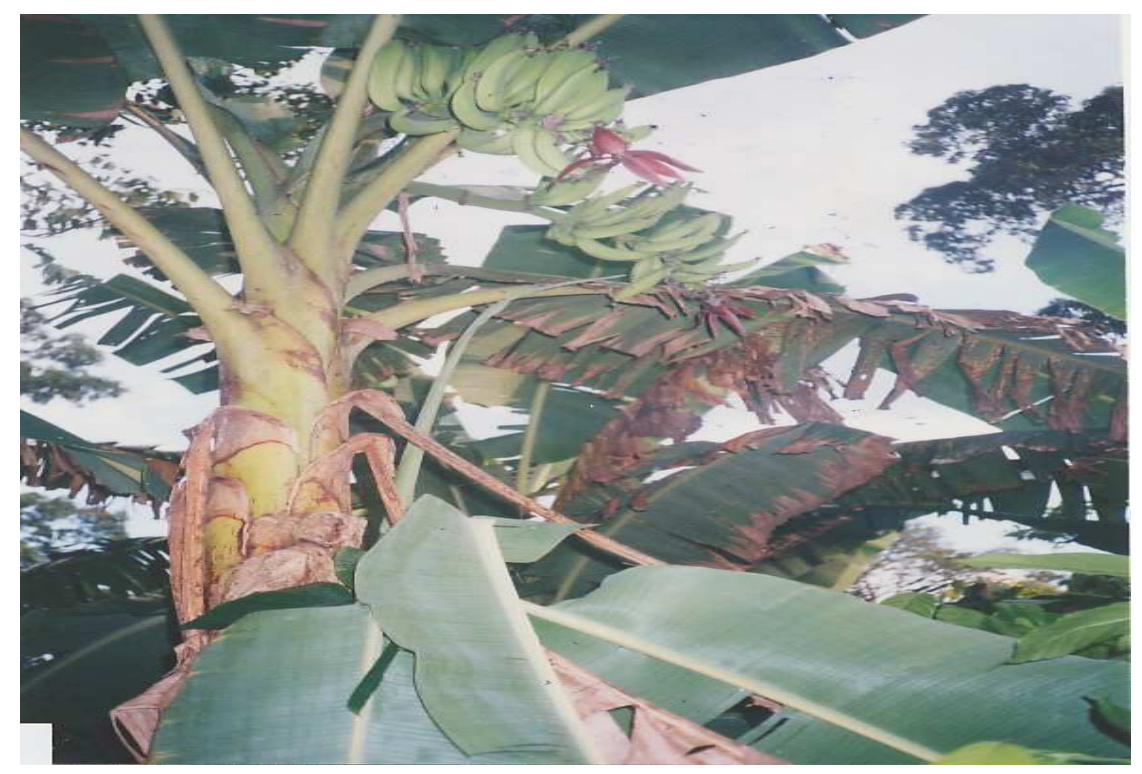

Figure 4 : Un pied de Taévé portant 2 régimes. 
Tableau 1 : Quelques caractéristiques des variétés de bananiers plantains recensés.

\begin{tabular}{|c|c|c|c|c|c|}
\hline Variétés & Agbavé & Apim & Kadaga & Taévé & Abladzo \\
\hline Pseudo-tronc & $\begin{array}{l}\text { Pseudo-tronc } \\
\text { grand, } 5 \text { à } 6 \mathrm{~m} \\
\text { Inhibition forte } \\
\text { des rejets }\end{array}$ & $\begin{array}{c}\text { Pseudo-tronc grand } \\
\text { robuste vers le bas, } 6 \text { à } 7 \\
\text { m ; Inhibition des rejets } \\
\text { moyenne }\end{array}$ & $\begin{array}{l}\text { Pseudo-tronc } \\
\text { grand, } 4 \text { à } 5 \text { m } \\
\text { Inhibition } \\
\text { moyenne des rejets }\end{array}$ & $\begin{array}{l}\text { Pseudo-tronc moyen, } \\
4 \mathrm{~m} \text { environ; } \\
\text { Inhibition moyenne des } \\
\text { rejets }\end{array}$ & $\begin{array}{l}\text { Pseudo-tronc vert un peu grand } \\
\text { et moyen, } 4 \text { à } 5 \mathrm{~m} \text { Inhibition des } \\
\text { rejets forte. }\end{array}$ \\
\hline Feuille/pétiole & $\begin{array}{c}\text { Feuilles grandes } \\
\text { Canal pétiolaire de la feuille III } \\
\text { peu ouvert, marges recourbées vers } \\
\text { l'intérieur }\end{array}$ & $\begin{array}{c}\text { Feuilles grandes } \\
\text { Canal pétiolaire de la } \\
\text { feuille III fermé ou très } \\
\text { peu ouvert }\end{array}$ & $\begin{array}{l}\text { Feuilles grandes et } \\
\text { moyennes } \\
\text { Canal pétiolaire de } \\
\text { la feuille III peu } \\
\text { ouvert }\end{array}$ & $\begin{array}{l}\text { Feuilles grandes } \\
\text { Canal pétiolaire III très } \\
\text { ouvert, tendance des } \\
\text { marges à couvrir le } \\
\text { canal }\end{array}$ & $\begin{array}{c}\text { Feuilles grandes } \\
\text { Canal pétiolaire de feuille III est } \\
+ \text { ou }- \text { fermé. }\end{array}$ \\
\hline Régime/Fruits & $\begin{array}{l}\text { Un régime avec } 2 \text { à } 3 \text { mains, une } \\
\text { main porte } 1 \text { à } 12 \text { doigts ; fruit } \\
\text { incurvé apex effilé Taille du fruit: } \\
28 \text { à } 30 \mathrm{~cm}\end{array}$ & $\begin{array}{c}\text { Régime en position } \\
\text { verticale au pseudo-tronc. } \\
\text { Un régime donne } 16 \\
\text { mains ; une main porte } 18 \\
\text { doigts ; } \\
\text { Taille du fruit : } 16 \text { à } 18 \\
\text { cm extrémité effilé ; peau } \\
\text { et pulpe du fruit mur : } \\
\text { jaune }\end{array}$ & $\begin{array}{l}\text { Régime en position } \\
\text { oblique sur la } \\
\text { plante. Un régime } \\
\text { donne } 6 \text { mains ; } \\
\text { une main porte } 6 \\
\text { doigts ; Taille du } \\
\text { fruit : } 20 \mathrm{~cm} \text {; fruit } \\
\text { incurvé sous forme } \\
\text { d'une pirogue ; } \\
\text { peau et pulpe jaune } \\
\text { à maturité }\end{array}$ & $\begin{array}{l}2 \text { régimes par pied ; } \\
\text { Un régime donne } 5 \\
\text { mains et une main } \\
\text { donne } 6 \text { doigts ou } \\
\text { fruits ; } \\
\text { Taille du fruit : } 15 \text { à } 20 \\
\text { cm, extrémité pointue, } \\
\text { fruit incurvé ; peau et } \\
\text { pulpe mur jaune. }\end{array}$ & $\begin{array}{l}\text { Régime petit court et ramassé ; } \\
\text { Un régime donne } 5 \text { mains et une } \\
\text { main porte } 10 \text { fruits de banane } \\
\text { Taille du fruit : } 16 \text { à } 18 \mathrm{~cm} \text {; } \\
\text { peau et pulpe de la banane jaune } \\
\text { à maturité. }\end{array}$ \\
\hline Utilisation du fruit & A cuire & A cuire & A cuire & A cuire & A cuire \\
\hline Cycle végétatif (mois) & 15 & 18 & 11 à 14 & 13 & 13 \\
\hline
\end{tabular}




\section{DISCUSSION}

La région des Plateaux Ouest du Togo est une zone propice pour la culture des bananiers à cause des meilleures conditions pédoclimatiques. Malgré ces conditions favorables, l'exploitation des bananiers plantains est confrontée à de nombreux défis qu'il est urgent de relever. En effet, la superficie cultivée par chaque agriculteur n'est pas connue. Le travail à la houe ne permet pas aux agriculteurs de cultiver des superficies dépassant un hectare. Ceci s'explique par les faibles quantités de bananes plantains produites (FAO, 2009). Nous n'avons pas de données significatives de la production pour évaluer aussi bien les pratiques culturales et les performances agronomiques de chaque variété pour apprécier les rendements des plantations. Il existe en plus des problèmes phytopathologiques qui perturbent la production des plantations et qui méritent d'être abordés pour des programmes d'amélioration de la culture du bananier comme l'a rapporté Daniells (2000).

L'identification des diverses variétés recensées a été faite en utilisant les descripteurs du bananier selon IPGRIINIBAP/CIRAD (1996); Tomékpé (1996) et Daniells et al. (2001). Mais, compte tenu de la forte variabilité phénotypique due souvent à l'environnement, cette description des différentes variétés est souvent difficile comme l'ont signalé Bakhiet et Elbadri (2004). Cependant, elle paraît intéressante pour faire dans un premier temps l'inventaire des variétés de bananiers cultivés grâce à une description agromorphologique. Cette description permet de faire une différenciation rapide et plus facile des divers phénotypes variétaux. Elle permet ensuite de connaître et de dénombrer les variétés locales de bananiers plantains cultivés pour évaluer ensuite leur productivité, leurs performances agronomiques et mieux prévoir leur gestion. La diversité génétique des bananiers en général mérite d'être maîtrisée pour envisager des études de production massive et entreprendre des techniques d'innovation et augmenter les rendements.

La production bananière est presque destinée à l'autoconsommation mais, elle reste insuffisante à cause de la faible production. En effet, la banane plantain sert à la fois d'aliment énergétique et de dessert. Elle joue un rôle socio-économico-culturel très important. La banane plantain sert à produire de la farine qui est utilisé dans la fabrication des beignets, biscuits et gâteaux (Kasongo, 2005). La banane a une composition chimique voisine à celle du mucus, tapissant l'estomac. Ainsi, elle joue un rôle dans le traitement des ulcères gastriques et la diarrhée. Et sa forte teneur en vitamine B6 aide à soulager le stress et l'anxiété (Morris et al., 2010). Aux EtatsUnis d'Amérique, un extrait naturel du pseudo tronc est commercialisable en tant que complément alimentaire et comme une pilule préventive contre le cancer. Les bananiers fournissent des fibres très utilisées dans la fabrication de certains papiers utilisés dans l'industrie de manufacturation des sachets de thé et des billets de banque au Japon. Et ces fibres ont d'autres usages dans la fabrication des cordes, ficelles et de nombreux objets d'artisanat (CIRAD, 2001). En outre, les feuilles de bananiers s'utilisent comme toiture lorsqu'elles sont séchées, comme couverture de contour des latrines, comme lit ou natte, comme porte objet sur la tête, comme assiette (Lassoudière, 2011), comme couvre aliment pendant la cuisson, comme emballage d'aliments, enfin comme papier hygiénique dans certaines circonstances.

L'importance et les valeurs nutritionnelles des bananes méritent qu'on leur accorde une attention toute particulière. La banane possède également des propriétés thérapeutiques comme la diminution de la pression sanguine, la prévention et la guérison des ulcères et des maladies cardiovasculaires (Valmayor et Dinh, 2002). Pour les sportifs, la banane est particulièrement indiquée, en raison de sa richesse en glucide, en vitamines $\mathrm{B}$, en potassium et en magnésium, qui sont des éléments minéraux très importations pour 
le fonctionnement musculaire. Elle participe au maintient des défenses immunitaires grâce à ces apports en vitamines $\mathrm{C}$ et $\mathrm{B}$, en minéraux et en oligoéléments variés (INIBAP, 2001; CIRAD-GRET, 2002 ; Honfo et al., 2007).

\section{Conclusion}

Cette étude préliminaire nous a permis de faire la prospection des variétés de bananiers plantains cultivés dans la zone Ouest de la Région des Plateaux au Togo et de mesurer leur importance dans l'alimentation locale. La diversité génétique au sein de ce groupe a été bien identifiée malgré leur apparence homogène. La banane plantain est une source alimentaire importante et ses bienfaits sur la santé ne sont plus à démontrer. Ainsi, il importe que des travaux soient entrepris pour la maîtrise de sa production. Des études sur la diversité génétique des bananiers, les performances agronomiques des variétés, les sols et les conditions climatiques, la phytopathologie, la caractérisation moléculaire et sur la sélection des variétés performantes méritent d'être entreprises. Des outils modernes de micropropagation tels que les biotechnologies peuvent permettre une multiplication plus rapide de milliers de plants de bananiers sains dans un temps court et dans un espace réduit et accroître la taille des plantations. L'augmentation des bananeraies avec des variétés sélectionnées hautement productives influencera ainsi positivement la production agricole locale pour mieux répondre aux besoins des populations et accrôitre la disponibilité alimentaire des bananes au Togo.

\section{REFERENCES}

Anirudhan TS, Shibi IG. 2007. Préparation d'un échangeur de cations contenant des groupements carboxyl issus de pseudotronc de bananier et son utilisation comme agent chélateur. InfoMusa, 16(12): 7-11.

Anonyme. 2013. Banane et banane plantain. http://www.passeportsante.net/fr/Nutritio n/ EncyclopedieAliments/Fiche.aspx?doc =banane_nu, Consulté le 06/09/2013.

Bakhiet SB, Elbadri AAG. 2004. Effet de la profondeur de la plantation sur la durée du cycle de production et le rendement. InfoMusa, 13(1): 12-14.

Bakry F, Didier C, Ganry J, Belliec F, Lescot T, Pinon A, Rey JY, Teisson C, Vanniere H. 2002. Plantes Fruitières in Memento de l'Agronome. CORD-GRET : 960-974.

Brabant P, Darracq S, Egue K, Simmonneaux V. 1996. Togo : Etat de Dégradation des Terres Résultant des Activités Humaines (Notice explicative de la carte). ORSTOM : Paris ; 57 p.

Champion J. 1963. Le Bananier. Ed. G-P Maisonneuve et La Rose : Paris ; 263p.

CIRAD. 2001. Les Usages Multiples du Bananier. CIRAD.

CIRAD-GRET. 2002. Mémento d'agronomie, centre de coopération international en recherche et échange technologique (GRET), imprimé en France Jouve, 11Bd de Sébastopol, dépôt légal, 191p.

Daniells J, Christophe J, Karamura D, Tomekpe K. 2001. Musalogue : A catalogue of Musa germplasm. Diversity in the genus Musa. (INIBAP/ CTA / CIRAD)

Daniells J. 2000. Quelle variété de bananier cultiver? InfoMusa, 9(1): 31-33.

FAO. 2009. Productions 2008, données de FAOSTAT, FAO.

Gire A. 1994. Relation entre la résistance partielle du bananier à Cercospora figiensis et une composante cellulaire constitutive de nature polyphénolique; Mémoire de (DEA), Laboratoire de biotechnologie et de physiologie appliquée, Université Montpellier II France, 14p.

Honfo F, Hell K, Coulibaly O, Tenkouano A. 2007. Teneurs en oligo-éléments et contribution des aliments dérivés de banane plantain aux apports journaliers en fer, zinc et $\beta$-carotène dans le sud du Nigeria. InfoMusa, 16(1-2): 1-6.

INIBAP. 2005. Rapport annuel, INIBAP, 5p. 
INIBAP. 2001. Annual rapport 2001. International network for improvement of banana and banana plantain, Montpelier, France, 42p.

IPGRI - INIBAP/CIRAD. 1996. Descripteurs pour le bananier (Musa spp). 55p.

Jones DR. 2000. Diseases of Banana, Abaca and Enset. CABI Publishing, CAB International: Royaume-Uni; 544p.

Kasongo K. 2005. Etude du taux des multiplications rapides Ex situ chez 3 cultivars acuminata diploïde, triploïde, tétraploïde de bananier après décapitation et utilisation de 6 benzylaminopyrine (BAP) à Kisangani, Mémoire, Faculté des sciences, UNIKIS, 39p.

Kone T, Kone M, Kone D, Kouakou TH, Traore S, Kouadio YJ. 2010. Effet de la photopériode et des vitamines sur la micropropagation du bananier plantain (Musa AAB) à partir de rejets écailles de rang1. Journal of Applied Biosciences, 26: 1675 - 1686.

Nkendah R, Akyeampong E. 2003. Données socioéconomiques sur la filière plantain en Afrique Centrale et de l'Ouest. InfoMusa, 12(1): 8-13.

Lassoudière A. 2011. Le bananier, un enjeu mondial. In Revue Pour la Science, 400: 78-85.

Lescot T. 2007. - Les dossiers de Fruitrop : la diversité génétique des bananiers. Fruitrop, 145: 28-35.

Morris MS, Sakakeeny L, Jacques PF, Picciano MF, Selhub J. 2010. Vitamin B6 intake is inversely related to, and the requirement is affected by, inflammation status. J. Nutr., 140(1): 103-310.
Odah K, Aziadekey G, Tozo K, Akpavi S, Koukouma R, Guelly A, Kokou K, Assignon K, Akogo Y, Aidam A, Akpagana K, Kenny L, Gbeassor M. 2012. Etude préliminaire de la diversité génétique du bananier (Musa spp.) et sa valeur dans l'alimentation au Togo. J. Rech. Sci. Univ., 14(2):119-127.

Perrier X, Tezenas DMH. 1990. Musaid: A computerize determination system. In Identification of Genetic Diversity in the Genus Musa, Persley CJ, de Langhe EA (Eds), proceeding of an international workshop held at los banos, Philippines, INIBA, Montferrier-Sur-Lez, France, pp.176-91.

Tomekpe Kodjo, Moïse Kwa, Beloved Mensah Dzomeku , Jacky Ganry. 2011. CARBAP and innovation on the plantain banana in Western and Central Africa. International J. Agr. Sust., 9(1): 264-273.

Tomekpe K. 1996. Caractérisation des Bananiers dans Recherches sur la gestion des cultures de bananes et de bananes plantain. Stage régional de formation, CRBP/IITA, pp. 17-29.

Valmayor RH, Dinh D. 2002. Les bananiers à cuire, classification, production et utilisations en Asie du Sud-Est. InfoMusa, 9(1): 28-30. 